Frederic Shapiro Navil Sethna

\section{Blood loss in pediatric spine surgery}

vertebral levels incorporated into the fusion, with posterior fusions compared to anterior fusions, and in those patients having both anterior and posterior fusions.

Keywords Scoliosis surgery · Pediatric age group $\cdot$ Blood loss
F. Shapiro ( $\bowtie)$

Department of Orthopaedic Surgery, Children's Hospital Boston,

300 Longwood Avenue,

Boston, MA 02115, USA

Tel.: +1-617-3556753,

Fax: +1-617-7300236,

e-mail:

frederic.shapiro@childrens.harvard.edu

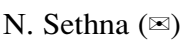

Department of Anesthesiology,

Perioperative and Pain Medicine,

Children's Hospital Boston,

300 Longwood Avenue, Boston,

MA 02115, USA

Tel.: +1-617-3554146,

Fax: +1-617-7300894,

e-mail:

navil.sethna@childrens.harvard.edu

\begin{abstract}
This article reviews the extent of blood loss in spine surgery for scoliosis corrections in the pediatric age group. An extensive literature review presents blood loss values in surgery for adolescent idiopathic scoliosis, cerebral palsy, Duchenne muscular dystrophy, spinal muscular atrophy, and myelomeningocoele. The underlying disorder plays a major role in determining the extent of blood loss. Blood loss is considerably higher in those patients with a neuromuscular scoliosis compared with adolescent idiopathic scoliosis. Within the neuromuscular group those with Duchenne muscular dystrophy demonstrate the highest mean levels of blood loss. Blood loss is also shown to be progressively greater with increasing numbers of
\end{abstract}

\section{Introduction}

Blood loss is an important concern in performance of spinal surgery. This article will review the extent of blood loss in spine surgery for scoliosis and kyphoscoliosis corrections in the pediatric age group since these are the procedures which are most extensive and subject to the greatest amounts of loss.

The pediatric age group refers to the first two decades of life, with the majority of spinal surgical procedures done in the second decade. Distinction must be made between studies in pediatric and adult patients, since blood loss in the adult patient can be proportionately greater than in the pediatric age group for the same procedure. The modern era of scoliosis surgery will be reviewed involving procedures performed from the early 1960s onward using spinal instrumentation and spine fusion for correction and stabilization of deformity.

The underlying disorder plays a major role in determining the extent of blood loss. Blood loss is considerably higher in those patients whose scoliosis is associated with a neuromuscular disease compared with those in the idiopathic category. Blood loss is also shown to be progressively greater with increasing numbers of vertebral levels incorporated into the fusion and with posterior fusions compared to anterior fusions. 


\section{Considerations regarding literature reports of blood loss in scoliosis surgery}

Blood loss determinations

Limitations must be recognized in the accuracy of intraoperative blood loss determinations and, therefore, in the value of comparing studies from one center to another. Blood loss is reported as "estimated blood loss" (EBL) since this represents the only practical way that operating room determinations can be made. Since these are estimates they are not rigidly accurate or reproducible as they are dependent on a combination of numbers including: volume of blood suctioned from the operative field (from which irrigating fluid must be subtracted), determination of blood loss collected on sponges (as determined by weighing by the operating room nurses), and estimates of blood loss on drapes, gowns, and floor (which is educated guesswork at best). To a great extent the value determined is dependent on the degree of rigor used by the operating room team in making the determination.

Measurement values used to present information

Different ways of presenting blood loss information are used in different papers reporting on scoliosis surgery. In almost all studies the extent of blood loss is reported in total milliliters ( $\mathrm{mL}$ or $\mathrm{ml}$ ) or cubic centimeters (cc). A second way is by determination of blood loss per vertebral level included in the fusion (total blood loss divided by number of vertebral levels in the fusion). A third way calculates the blood loss as a percentage in relation to the patient's estimated blood volume (EBV). The EBV is generally calculated to be $70 \mathrm{ml} / \mathrm{kg}$ (weight) [21]. For a $50 \mathrm{~kg}$ patient the EBV is $70 \times 50=3,500 \mathrm{ml}$. A blood loss of $1,000 \mathrm{ml}$ in that patient would represent a $28.6 \%$ loss. Wider presentation of blood loss as percent EBV would be helpful since this value provides the most physiologic indicator by taking patient size into account.

\section{Diagnostic category of scoliosis}

Awareness of the disorder associated with the scoliosis is essential since distinction must be made between idiopathic scoliosis and secondary scoliosis, which usually refers to neuromuscular disorders causing the scoliosis. Some reviews lump all types of neuromuscular deformity together but in many reports they are subdivided into the common variants of neuromuscular scoliosis which are cerebral palsy, myelomenigocoele, and Duchenne muscular dystrophy (DMD). Each of these disorders has differing responses regarding blood loss during surgery as well as differing needs for spinal correction and stabilization.
Types of surgical procedure

Distinction is made regarding blood loss in surgery between solitary posterior spinal fusions, solitary anterior spinal fusions, and combined anterior and posterior spinal fusions (including consideration of whether the anteriorposterior procedures are done as a single operation or separately with 1-2 weeks between stages).

Primary reason for the report

from which blood loss information is abstracted

A few papers in this review were directed specifically to determining blood loss, usually comparing one anesthetic method to another or the use of a particular blood loss reducing agent. Since this article provides an overview of the problem of blood loss in pediatric spine fusion surgery, we have used the control group values from such studies but have usually also presented findings with the particular modification used.

Since limiting intra-operative blood loss is universally recognized as highly important, essentially all procedures done since the 1970s have incorporated several control mechanisms without necessarily indicating so in reports. These include: minimizing abdominal pressure with positioning, some form of hypotensive anesthesia, blood transfusion at pre-determined levels of hemoglobin, early replacement of platelets and fibrinogen, and surgical techniques stressing rigorous mechanical wound hemostasis [21, 26, 47, 57, 67]. For these reasons, most comparative studies relating to methods diminishing intra-operative blood loss are not scientifically controlled.

The large majority of papers in this review addressed problems relating to surgical technique, stabilization systems, and concerns specific to particular disorders and types of scoliosis. We have abstracted blood loss data as presented in each paper and derived EBL/vertebral segment and percent EBV numbers if sufficient information was provided.

The paper is designed to review intra-operative blood loss data. Some reports also assess post-operative blood loss but usually as separate determinations. In the few instances where combined data only were provided we have indicated that fact, and have used such studies only if the author indicated that post-operative loss was negligible.

\section{Review of blood loss data from the literature}

Blood loss data from the literature are presented in Tables 1, $2,3,4,5$ and 6 . In each table we have listed the study (article) with year of publication and year(s) when surgery was performed, estimated mean blood loss ( $\mathrm{ml}$ or $\mathrm{cc}$ ) with ranges and/or standard deviations, the number of operative procedures in the study, surgical technique with bone 
Table 1 Blood loss data from studies on posterior spinal fusions for AIS. $C$ - $D$ Cotrel-Dubousset (instrumentation), $H$-rod Harrington rod, TSRH Texas Scottish Rite Hospital (instrumentation), L-rod Luque rod

\begin{tabular}{|c|c|c|c|c|c|c|}
\hline $\begin{array}{l}\text { Study [reference] } \\
\text { (date of publication)/ } \\
\text { (date(s) of surgery) }\end{array}$ & $\begin{array}{l}\text { Estimated blood } \\
\text { loss (EBL) (ml) } \\
{[\text { mean (range)] }}\end{array}$ & $\begin{array}{l}\text { Number } \\
\text { of proce- } \\
\text { dures }\end{array}$ & Technique & $\begin{array}{l}\text { Estimated } \\
\text { blood loss/ } \\
\text { individual } \\
\text { vertebral level } \\
\text { in fusion (ml) } \\
\text { (EBL/\# } \\
\text { levels=) }\end{array}$ & $\begin{array}{l}\text { Estimated } \\
\text { blood loss/ } \\
\text { total } \\
\text { estimated } \\
\text { blood volume } \\
\text { (EBL/EBV) }\end{array}$ & Comments \\
\hline
\end{tabular}

\begin{tabular}{|c|c|c|c|}
\hline $\begin{array}{l}\text { Shufflebarger } \\
\text { et al. [62] } \\
(2004) /(1998)\end{array}$ & $\begin{array}{l}500(200-800) \\
627(350-1500) \\
675(500-750)\end{array}$ & $\begin{array}{l}55(\text { Lenke } 5) \\
7(\text { Lenke } 6) \\
3(\text { Lenke } 3 \mathrm{c})\end{array}$ & $\begin{array}{l}\text { Posterior shortening, } \\
\text { posterior segmental } \\
\text { pedicle screws with } \\
5 \mathrm{~mm} \text { rods } \\
\text { (Moss-Miami) }\end{array}$ \\
\hline $\begin{array}{l}\text { DuToit et al. [18] } \\
(1978) /\end{array}$ & 567 & 27 & $\begin{array}{l}\text { Harrington } \\
\text { rod }\end{array}$ \\
\hline
\end{tabular}

Copley et al. [16]

(1999)/(1995-1997)

608 (hemodilution) 43

672 (control) 43
Posterior fusions, systems not described

-
-
-

$\begin{array}{ll}608 / 10.7=57 & 608 / 3780=16 \% \\ 672 / 10.2=66 & 672 / 3774=18 \%\end{array}$
Guidera et al. [32] (1993)/

Erwin et al. [20]

(1976)/(1966-1972)

Harrington and

Dickson [33]

$(1973) /(1961-1972)$

Youngman and Edgar [71] (1985)/ (1974-1982)

Lawhon et al. [39]

(1984)/(1972-1978)
743

840

$748(110-3480)$

$779(<200->1400)$

801

\footnotetext{
Siller et al. [63] (1996)/(1991)

Florentino-Pineda et al. [26] (2004)/ (1999-2001)
}

\begin{tabular}{|c|c|c|}
\hline 1583 & 31 & $\begin{array}{l}\text { Harrington rod } \\
\text { technique }\end{array}$ \\
\hline $823(186-1587)$ & 55 & $\begin{array}{l}\text { C-D, iliac crest } \\
\text { bone graft }\end{array}$ \\
\hline $893+/-220$ & 19 & $\begin{array}{l}\text { Posterior spinal fusion } \\
\text { with segmental }\end{array}$ \\
\hline $952+/-372$ & 17 & $\begin{array}{l}\text { instrumentation; } \\
\text { allograft only }\end{array}$ \\
\hline
\end{tabular}

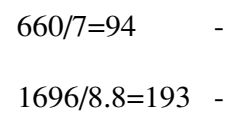

Harrington-rod, no iliac crest bone graft Harrington rod, iliac crest bone graft

Harrington rod, iliac crest bone graft

Harrington rod, iliac crest bone graft, multiple ancillary procedures (operation in cast, sublaminar wires, etc)

Harrington rod technique

1084/11.1=98 -

$893 / 12=74$

$952 / 12=79$
No iliac crest graft

-
-
-
$608 / 3780=16 \%$
$672 / 3774=18 \%$

Use of acute hemodilutional autotransfusion intraoperatively

Comparison of hemodilution technique to control group, iliac crest bone graft, multicenter

Smaller curves $<60^{\circ}$, levels $=7$ Larger curves $>60^{\circ}$, levels $=8.8$ [numbers per group not listed]

-

Hypotensive anesthesia, systolic $85-95 \mathrm{mmHg}$ Induced hypotension, systolic 60-65 mmHg

Induced hypotension, mean arterial pressure (diastolic pressure $+1 / 3$ of pulse pressure) less than $90 \mathrm{mmHg}$ Normotensive anesthesia

Hypotensive anesthesia

$893 / 3095=29 \% \quad \varepsilon$-aminocaproic acid group

$952 / 3153=30 \% \quad$ Control group 
Table 1 (continued)

\begin{tabular}{|c|c|c|c|c|c|c|}
\hline $\begin{array}{l}\text { Study [reference] } \\
\text { (date of publication)/ } \\
\text { (date(s) of surgery) }\end{array}$ & $\begin{array}{l}\text { Estimated blood } \\
\text { loss (EBL) (ml) } \\
{[\text { mean (range) }]}\end{array}$ & $\begin{array}{l}\text { Number } \\
\text { of proce- } \\
\text { dures }\end{array}$ & Technique & $\begin{array}{l}\text { Estimated } \\
\text { blood loss/ } \\
\text { individual } \\
\text { vertebral level } \\
\text { in fusion (ml) } \\
\text { (EBL/\# } \\
\text { levels=) }\end{array}$ & $\begin{array}{l}\text { Estimated } \\
\text { blood loss/ } \\
\text { total } \\
\text { estimated } \\
\text { blood volume } \\
\text { (EBL/EBV) }\end{array}$ & Comments \\
\hline
\end{tabular}

Florentino-Pineda et al. [25] (2001)/

$988(+/-411)$

29 (1996-1998)

$\begin{array}{lll} & 1405(+/-671) & 3 \\ \text { Patel et al. [56] } & 1102(+/-72) & 27 \\ (1985) /(1977-1980) & 1541(+/-156) & 22\end{array}$

Moran et al. [48]

1113

(1995)/(1989-1993)

Richards et al. [59]

(1994)/(1988-1991)

Guadagni et al. [30]

(1984)/(1979-1982)

McMaster [46]

(1991)/(1975-1987)

Lenke et al. [40]

(1992)/(1985-1988)

Albers et al. [1]

(2000)/(1991-1995)

$1421(+/-881)$

21

$1801(+/-1201)$

24

Lovallo et al. [42]

(1986)/(1978-1982)

Barr et al. [3]

(1997)/

Guay et al. [31]

$1971(+/-831)$

30

31

27

22

84

95

30

31

156

152

95

$1500(300-4000)$

$1571(550-3300)$

39

(1994)/
Multiple

instrumentations

$988 / 12=82$
C-D, iliac crest

bone graft

TSRH

H-rod, L-rod with spinous process wiring H-rod

H-rod

L-rod

C-D, autogenous

bone graft iliac crest

(occasional ribs)

Single rod TSRH,

11 iliac crest bone graft

Dual rods (TSRH,

C-D or Paragon)

17 iliac crest bone graft

H-rod, autogenous iliac crest bone graft

C-D (thoracic) but lumbar pedicle hooks/ screws 20 and lumbar hooks only 19

C-D, autogenous iliac crest bone graft

988/2973=33\% Pre-operative autologous blood and controlled hypotension (systolic $20 \%$ less than preinduction value) $\varepsilon$-aminocaproic acid (EACA)

No EACA

$\begin{array}{ll}1405 / 12= & 1405 / 3188= \\ 117 & 44 \%\end{array} \quad$ No EACA

117

46

84

$1102 / 3836=$ $29 \%$

$1541 / 4186=$ $37 \%$

$1187 / 11=108-$ $1543 / 10=154$

$1200 / 11+109^{a}-$ $1490 / 10.9=\quad-$ $137^{\text {a }}$

$1211 / 11=110 \quad-$

$1500 / 10=150 \quad-$

$1571 / \sim 10=157-$

$1971 / 9.3=212 \quad 61.5 \%$

$1971 / 9.3=212.61 .5 \%$

Induced moderate hypotensive anesthesia (Systolic blood pressure 20-30 mmHg < preoperative systolic pressure)

Preoperative autologous blood donation

Same surgeon all cases, Autogenous iliac crest grafts

Double major curves; blood loss same in two approaches

Low normotensive anesthesia

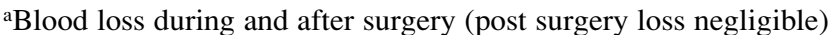


Table 2 Blood loss data from studies on anterior spinal fusions for AIS. TSHR Texas Scottish Rite Hospital (instrumentation), $C$ - $D$ Cotrel-Duboussett

\begin{tabular}{|c|c|c|c|c|c|c|}
\hline $\begin{array}{l}\text { Study [reference] } \\
\text { (date of publication) / } \\
\text { (date(s) of surgery) }\end{array}$ & $\begin{array}{l}\text { Estimated } \\
\text { blood loss } \\
(\text { EBL })(\mathrm{ml}) \\
{[\text { mean (range)] }}\end{array}$ & $\begin{array}{l}\text { Number } \\
\text { of } \\
\text { proce- } \\
\text { dures }\end{array}$ & Technique & $\begin{array}{l}\text { Estimated blood } \\
\text { loss / individual } \\
\text { vertebral level in } \\
\text { fusion (ml) } \\
\text { (EBL/\# levels=) }\end{array}$ & $\begin{array}{l}\text { Estimated } \\
\text { blood loss/ } \\
\text { total blood } \\
\text { volume }\end{array}$ & Comments \\
\hline $\begin{array}{l}\text { Moskowitz, } \\
\text { Trommanhauser [49] } \\
(1993) /(1983-1989)\end{array}$ & 330 & 13 & Zielke, rib graft & $330 / 4.4=75$ & - & - \\
\hline $\begin{array}{l}\text { Bernstein, Hall [9] } \\
(1998) /(1990-1993)\end{array}$ & 344 & 17 & TSRH (anterior) & $344 / 3.4=101$ & - & - \\
\hline $\begin{array}{l}\text { Turi et al. [69] } \\
(1993) /\end{array}$ & $401(100-800)$ & 14 & TSRH (anterior), rib graft & $401 / 5=80$ & - & - \\
\hline \multirow[t]{2}{*}{$\begin{array}{l}\text { Newton et al. [52] } \\
(2003) /(1991-2001)\end{array}$} & $\begin{array}{l}424 \\
(+/-302)\end{array}$ & 38 & $\begin{array}{l}\text { Thoracoscopic anterior } \\
\text { instrumentation Isolated } \\
\text { structural thoracic curves- } \\
\text { Lenke IA, IB, IC }\end{array}$ & $424 / 7=61$ & - & \multirow{2}{*}{$\begin{array}{l}\text { An earlier } \\
\text { study compared } \\
\text { thorascopic } \\
\text { anterior release } \\
\text { and fusion (EBL } \\
235 \mathrm{ml} \text { ) with } \\
\text { fusion by open } \\
\text { thoracotomy } \\
\text { (EBL } 270 \mathrm{ml} \text { ) } \\
\text { (reference 53) }\end{array}$} \\
\hline & $551(+/-363)$ & 68 & $\begin{array}{l}\text { Anterior open instrumen- } \\
\text { tation (Depuy-Acro Med } \\
\text { Harms Study group) }\end{array}$ & - & - & \\
\hline $\begin{array}{l}\text { Bullman et al. [14] } \\
\text { 2003/ }\end{array}$ & $\begin{array}{l}437 \\
(+/-221 \\
100-1000)\end{array}$ & 45 & $\begin{array}{l}\text { Anterior dual rod Halm- } \\
\text { Zielke }\end{array}$ & $437 / 4.7=93$ & - & - \\
\hline Bitan et al. [11] 2003/ & $\begin{array}{l}505 \\
(150-1000)\end{array}$ & 24 & $\begin{array}{l}\text { TSRH, Moss-Miami and } \\
\text { C-D Horizon rib graft }\end{array}$ & $505 / 2.9=174$ & - & - \\
\hline $\begin{array}{l}\text { Majad et al. [44] } \\
(2000) /\end{array}$ & $590(250-950)$ & 22 & $\begin{array}{l}\text { TSRH, Moss-Miami, Isola/ } \\
\text { rod (3-11 levels fused) }\end{array}$ & - & - & - \\
\hline $\begin{array}{l}\text { Lowe, Peters [43] } \\
1993 /\end{array}$ & 610 & 36 & Zielke, rib graft & $610 / 4.5=136$ & - & - \\
\hline $\begin{array}{l}\text { Hopf et al. [35] } \\
1997 /(1992-1994)\end{array}$ & $\begin{array}{l}630 \\
(400-1200)\end{array}$ & 16 & $\begin{array}{l}\text { C+D - Hopf anterior } \\
\text { system }\end{array}$ & - & - & - \\
\hline $\begin{array}{l}\text { Kaneda et al. [37] } \\
\text { 1997/(1992-1994) }\end{array}$ & 650 & 20 & $\begin{array}{l}\text { Kaneda anterior spinal } \\
\text { system }\end{array}$ & $650 / 7.5=87$ & - & - \\
\hline $\begin{array}{l}\text { Betz et al. [10] } \\
(1999) /(1991-)\end{array}$ & $\begin{array}{l}956 \\
(+/-857)\end{array}$ & 78 & $\begin{array}{l}\text { Flexible rods (Harms-Moss, } \\
\text { DuPuy-Motech-Acromed) }\end{array}$ & - & - & - \\
\hline $\begin{array}{l}\text { Hsu et al. [36] } \\
(1982) /\end{array}$ & $\begin{array}{l}1645 \\
(440-3400)\end{array}$ & 28 & Dwyer & $1645 / 4.7=350$ & - & - \\
\hline
\end{tabular}

graft information, mean EBL per individual vertebral level in fusion, mean EBL as a percentage of total EBV, and additional comments. In each table the studies are listed in order of mean EBL, beginning with those with the least loss reported to those with the highest amounts.

Table 1 reviews posterior spinal fusions for adolescent idiopathic scoliosis (AIS), Table 2 reviews anterior spinal fusions for AIS, Table 3 reviews spinal fusions for cerebral palsy, Table 4 reviews posterior spinal fusions for DMD, Table 5 reviews spinal fusions for pooled neuromuscular disorders (both anterior and posterior approaches), and Table 6 reviews spinal fusions for other neuromuscular disorders including spinal muscular atrophy and myelomeningocoele.

\section{Blood loss in scoliosis surgery assessed by underlying disorder}

Awareness of potential blood loss problem with any spinal fusion surgery

The operating team must have a high degree of preparation for blood loss in any spinal surgery for scoliosis in the pediatric age group, regardless of etiology and surgical approach to correction. Although AIS treated by spinal fusion has the lowest mean values for blood loss in scoliosis procedures, the ranges of blood loss measurements in all studies are wide, and amounts necessitating blood transfusion are often noted for both anterior and posterior approaches (Tables 1 and 2). 
Table 3 Blood loss data from studies on spinal fusions for cerebral palsy. $P$ posterior spinal fusion, $A$ anterior spinal fusion, $P / a$ posterior spinal fusion with some cases having anterior spinal fusion, $H$-rod Harrington rod system, L-rod Luque rod system, $D D A V P$ desmopressin acetate

\begin{tabular}{|c|c|c|c|c|c|c|}
\hline $\begin{array}{l}\text { Study [reference] } \\
\text { (date of publication) / } \\
\text { (date(s) of surgery) }\end{array}$ & $\begin{array}{l}\text { Estimated } \\
\text { blood loss } \\
(\text { EBL })(\mathrm{ml}) \\
{[\text { mean (range)] }}\end{array}$ & $\begin{array}{l}\text { Number } \\
\text { of pro- } \\
\text { cedures }\end{array}$ & Technique & $\begin{array}{l}\text { Estimated blood } \\
\text { loss / individual } \\
\text { vertebral level } \\
\text { in fusion }(\mathrm{ml}) \\
(\mathrm{EBL} / \# \text { levels=) }\end{array}$ & $\begin{array}{l}\text { Estimated } \\
\text { blood loss / } \\
\text { total blood } \\
\text { volume }\end{array}$ & Comments \\
\hline \multirow[t]{2}{*}{$\begin{array}{l}\text { Bulman et al. [15] } \\
1996 /(1988-1993)\end{array}$} & $\begin{array}{l}\mathrm{P} / \mathrm{a} 1325 \\
(350-4000)\end{array}$ & 15 & $\begin{array}{l}\text { Luque-Galveston (15) } \\
\text { plus anterior release, disk } \\
\text { excision, arthrodesis ( } 7 \text { ) }\end{array}$ & $1325 / 14.5=91$ & - & \multirow[t]{2}{*}{$\begin{array}{l}\text { Same day proce- } \\
\text { dures; blood loss } \\
\text { for total approach }\end{array}$} \\
\hline & $\begin{array}{l}\mathrm{P} / \mathrm{a} 1240 \\
(300-3400)\end{array}$ & 15 & $\begin{array}{l}\text { Unit rod (15) plus } \\
\text { anterior release, disk } \\
\text { excision, arthrodesis (4) }\end{array}$ & $1240 / 15=83$ & - & \\
\hline \multirow[t]{2}{*}{$\begin{array}{l}\text { Sponseller et al. [64] } \\
\text { 1986/1982-1984 }\end{array}$} & $\begin{array}{l}\text { P } 1681 \\
\text { (hypotensive } \\
\text { anesthesia) }\end{array}$ & 20 & \multirow{2}{*}{$\begin{array}{l}\text { H-rod/L-rod, } 1 \text { or } 2 \text { with } \\
\text { spinous process wiring, } \\
\text { iliac crest bone graft; } \\
\text { anterior release } 2 \text { weeks } \\
\text { pre posterior fusion in } \\
21-13 \text { with Dwyer } \\
\text { instrumentation }\end{array}$} & $1681 / 15=112$ & - & \multirow[t]{2}{*}{$\begin{array}{l}\text { Blood loss only } \\
\text { for posterior } \\
\text { procedures }\end{array}$} \\
\hline & $\begin{array}{l}\text { P } 2200 \\
\text { (normotensive } \\
\text { anesthesia) }\end{array}$ & 14 & & $2200 / 15=147$ & - & \\
\hline \multirow[t]{3}{*}{$\begin{array}{l}\text { Swank et al. [66] } \\
1989 /(1981-1985)\end{array}$} & $\begin{array}{l}\text { P } 1760 \\
(600-3000)\end{array}$ & 10 & $\begin{array}{l}\text { Luque or Luque- } \\
\text { Galveston, autogenous } \\
\text { iliac crest bone graft }\end{array}$ & $1760 / 12=147$ & - & \\
\hline & $\begin{array}{l}\text { A 906 } \\
(200-3600)\end{array}$ & 21 & $\begin{array}{l}\text { Anterior fusion, Zielke } \\
\text { system }\end{array}$ & $906 / 5=181$ & - & \multirow{2}{*}{$\begin{array}{l}\text { Two week } \\
\text { interval between } \\
\text { anterior and } \\
\text { posterior stages }\end{array}$} \\
\hline & $\begin{array}{l}\text { P } 2040 \\
(600-6490)\end{array}$ & - & $\begin{array}{l}\text { Posterior fusion, Luque } \\
\text { system, autogenous iliac } \\
\text { crest bone graft }\end{array}$ & $2040 / 14=146$ & - & \\
\hline \multirow[t]{2}{*}{$\begin{array}{l}\text { Allen, Ferguson [2] } \\
1982 /(1977-1980)\end{array}$} & $\begin{array}{l}\text { P } 2086 \\
(550-3900, \\
\text { both })\end{array}$ & 7 & $\begin{array}{l}\text { Luque rods to L5 or } \\
\text { above }\end{array}$ & $2086 / 10.7=195$ & - & \multirow[t]{2}{*}{-} \\
\hline & 2267 & 3 & $\begin{array}{l}\text { Luque rods to pelvis. } \\
\text { Both with iliac crest } \\
\text { autografts }\end{array}$ & $2267 / 14.7=154$ & - & \\
\hline $\begin{array}{l}\text { Gersoff, Renshaw } \\
{[28] 1988 /} \\
(1979-1983)\end{array}$ & P 2125 & 33 & $\begin{array}{l}\text { Luque rod, bone bank } \\
\text { bone graft }\end{array}$ & $2125 / 14=152$ & - & $\begin{array}{l}\text { Hypotensive } \\
\text { anesthesia not } \\
\text { used. No anterior } \\
\text { approaches }\end{array}$ \\
\hline \multirow{4}{*}{$\begin{array}{l}\text { Lonstein, Akbarnia } \\
\text { [41] 1983/ } \\
(1948-1979)\end{array}$} & I: A 1919 & 3 & & $1919 / 7=274$ & $\begin{array}{l}58 \% \mathrm{~EB} \\
\text { volume }\end{array}$ & \multirow[t]{2}{*}{$\begin{array}{l}\text { Group I - double } \\
\text { balances curves }\end{array}$} \\
\hline & P 2215 & 41 & $\begin{array}{l}\text { Harrington system, } \\
\text { autogenous iliac crest } \\
\text { bone graft }\end{array}$ & $2215 / 13=170$ & $\begin{array}{l}79 \% \mathrm{~EB} \\
\text { volume }\end{array}$ & \\
\hline & II: A 1803 & $>25$ & Dwyer or Zielke systems & $1803 / 7=258$ & $\begin{array}{l}54 \% \mathrm{~EB} \\
\text { volume }\end{array}$ & $\begin{array}{l}\text { Group II - large } \\
\text { unbalanced } \\
\text { thoraco-lumbra or } \\
\text { lumbar curves }\end{array}$ \\
\hline & P 2629 & 99 & $\begin{array}{l}\text { Harrington system, } \\
\text { multiple other methods }\end{array}$ & $2629 / 15=175$ & $\begin{array}{l}84 \% \mathrm{~EB} \\
\text { volume }\end{array}$ & $\begin{array}{l}\text { Some posterior } \\
\text { fusions without } \\
\text { instrumentation }\end{array}$ \\
\hline \multirow[t]{2}{*}{$\begin{array}{l}\text { Bonnett et al. [13] } \\
(1976) /(1960-1972)\end{array}$} & P 2230 & 10 & Harrington rod system & $2230 / 7.4=301$ & - & \multirow{2}{*}{$\begin{array}{l}\text { Many revisions } \\
\text { subsequently } \\
\text { needed with both } \\
\text { approaches. } \\
\text { Eventually } \\
\text { recommended } \\
\text { both anterior and } \\
\text { posterior- } \\
\text { improved results. }\end{array}$} \\
\hline & A 1500 & 18 & $\begin{array}{l}\text { Dwyer anterior fusion } \\
\text { system only }\end{array}$ & $1500 / 5.5=273$ & - & \\
\hline
\end{tabular}


Table 3 (continued)

\begin{tabular}{|c|c|c|c|c|c|c|}
\hline $\begin{array}{l}\text { Study [reference] } \\
\text { (date of publication) / } \\
\text { (date(s) of surgery) }\end{array}$ & $\begin{array}{l}\text { Estimated } \\
\text { blood loss } \\
(\text { EBL })(\mathrm{ml}) \\
{[\text { mean (range)] }}\end{array}$ & $\begin{array}{l}\text { Number } \\
\text { of pro- } \\
\text { cedures }\end{array}$ & Technique & $\begin{array}{l}\text { Estimated blood } \\
\text { loss / individual } \\
\text { vertebral level } \\
\text { in fusion }(\mathrm{ml}) \\
(\text { EBL/\# levels=) }\end{array}$ & $\begin{array}{l}\text { Estimated } \\
\text { blood loss / } \\
\text { total blood } \\
\text { volume }\end{array}$ & Comments \\
\hline $\begin{array}{l}\text { Dias et al. [17] } \\
(1996) /(1988-1991)\end{array}$ & P 2149 & 31 & $\begin{array}{l}\text { Unit rod to pelvis local } \\
\text { autogenous bone graft } \\
\text { plus bone back allograft. } \\
\text { T1 to sacrum }\end{array}$ & $2149 / 17=126$ & - & $\begin{array}{l}7 \text { patients had } \\
\text { anterior release } \\
\text { with rib graft } 1 \\
\text { week before } \\
\text { posterior fusion }\end{array}$ \\
\hline \multirow[t]{4}{*}{$\begin{array}{l}\text { Theroux et al. [68] } \\
(1997) /\end{array}$} & \multirow[t]{4}{*}{ Not reported } & \multirow[t]{4}{*}{21} & \multirow[t]{4}{*}{$\begin{array}{l}\text { Unit rod fusion } \mathrm{T} 1 \text { to } \\
\text { sacrum }\end{array}$} & \multirow[t]{4}{*}{-} & $\begin{array}{l}\text { DDAVP } \\
\text { group }\end{array}$ & \multirow[t]{4}{*}{-} \\
\hline & & & & & $\begin{array}{l}\text { Median loss } \\
148 \% \text { (range } \\
57-425) \text { EBV }\end{array}$ & \\
\hline & & & & & Placebo group & \\
\hline & & & & & $\begin{array}{l}\text { Median loss } \\
111 \% \text { (range } \\
65-240) \text { EBV }\end{array}$ & \\
\hline
\end{tabular}

Adolescent idiopathic scoliosis

AIS patients have the lowest amount of mean blood loss per procedure of all operative scoliosis groups. In the group treated by posterior spinal fusion (Table 1 ) in several studies the mean EBL ranges between 600 and $1,000 \mathrm{ml}$, and most studies document mean ranges between 750 and $1,500 \mathrm{ml}$. Treatment of AIS by anterior spinal fusion (Table 2) yields even lower mean EBL values, with most studies ranging between 350 and $650 \mathrm{ml}$ and almost all less than $1,000 \mathrm{ml}$.

While the ranges of EBL per vertebral level included in the fusion in AIS are similar for posterior approaches $(65-150 \mathrm{ml})$ and anterior approaches $(60-135 \mathrm{ml})$, the overall blood loss is considerably less in the anterior group because fewer levels need to be fused to gain acceptable correction. The number of levels fused in the anterior surgery group is usually between 4 and 7 while the posterior group involves 9 to 12 (Tables 1 and 2).

\section{Cerebral palsy}

Blood loss is considerably higher in cerebral palsy patients undergoing scoliosis correction compared to patients with AIS (Table 3). The mean blood loss ranges in posterior approaches are concentrated between 1,300 and $2,200 \mathrm{ml}$, while many in the anterior group range from 900 to $1,800 \mathrm{ml}$. Blood loss per vertebral level fused is approximately similar for anterior and posterior approaches, although greater than AIS amounts, with most studies reporting losses between 100 and $190 \mathrm{ml}$ per level. Blood loss amounts are much greater in posterior approaches primarily because of the larger number of vertebral levels involved; $13-15$ with posterior procedures and 5-7 with anterior procedures.

Duchenne muscular dystrophy

Blood loss in DMD patients is even higher in most series than amounts reported for cerebral palsy patients. Several large studies have reported mean blood loss amounts from $2,500 \mathrm{ml}$ to $4,000 \mathrm{ml}+$ (Table 4 ). Other studies have reported lower mean values of $930-1,680 \mathrm{ml}$ but even these reports show some patients at the $3,000,4,000 \mathrm{ml}$ levels. The blood loss amounts are further magnified in effect since many DMD patients are small in stature with low body weight. Fusion is invariably performed from the upper thoracic region to the sacrum encompassing 13-16 levels. Mean values of EBL per vertebral level are in the $200-280 \mathrm{ml}$ range, although more recent studies with less blood loss are being reported.

Studies on pooled neuromuscular disorders

Many studies on scoliosis surgery pool data from patients with neuromuscular disorders and do not distinguish between cerebral palsy, DMD, etc. Much valuable information is still provided, especially concerning blood loss with varying approaches. Most of these papers involve complex, severe deformities in which individual patients are treated by both anterior and posterior approaches. Mean EBL levels for anterior procedures are around $1,000 \mathrm{ml}$ but posterior approaches range from 2,000 to $3,500 \mathrm{ml}$. The EBL per individual vertebral level in the fusion is correspondingly high as well. 
Table 4 Blood loss data from studies on posterior spinal fusion for DMD. CDI Cotrel-Dubousset instrumentation, TSRH Texas Scottish Rite Hospital (instrumentation), ISOLA ISOLA instrumentation (Acromed)

\begin{tabular}{|c|c|c|c|c|c|c|}
\hline $\begin{array}{l}\text { Study [reference] } \\
\text { (date of publication) / } \\
\text { (date(s) of surgery) }\end{array}$ & $\begin{array}{l}\text { Estimated } \\
\text { blood loss } \\
(\text { EBL) }(\mathrm{ml}) \\
{[\text { mean (range)] }}\end{array}$ & $\begin{array}{l}\text { Number } \\
\text { of pro- } \\
\text { cedures }\end{array}$ & Technique & $\begin{array}{l}\text { Estimated blood } \\
\text { loss / individual } \\
\text { vertebral level in } \\
\text { fusion (ml) } \\
\text { (EBL/\# levels=) }\end{array}$ & $\begin{array}{l}\text { Estimated } \\
\text { blood loss / } \\
\text { total blood } \\
\text { volume }\end{array}$ & Comments \\
\hline $\begin{array}{l}\text { Marchesi et al. [45] } \\
(1997) /(1988-1993)\end{array}$ & $\begin{array}{l}930 \\
(750-1500)\end{array}$ & 25 & $\begin{array}{l}\text { Luque-Galveston with } \\
\text { sacral screws, local } \\
\text { bone graft plus } \\
\text { allogeneic bone }\end{array}$ & - & - & - \\
\hline $\begin{array}{l}\text { Fox et al. [27] } \\
(1997) /(1989-1994)\end{array}$ & $\begin{array}{l}1028 \\
(400-3000)\end{array}$ & 19 & $\begin{array}{l}\text { Hartshill rectangle, } \\
\text { allograft bone graft }\end{array}$ & $1028 / 12.5=82$ & $30 \%$ & $\begin{array}{l}\text { Hypotensive } \\
\text { anesthesia }\end{array}$ \\
\hline $\begin{array}{l}\text { Mubarak et al. [50] } \\
(1993) /(1980-1987)\end{array}$ & $\begin{array}{l}1680 \\
(250-4000)\end{array}$ & 22 & $\begin{array}{l}\text { Luque system (10), } \\
\text { Luque-Galveston } \\
\text { (to pelvis, 12) } \\
\text { autogenous iliac crest }\end{array}$ & $168 / 15.5=108$ & - & - \\
\hline $\begin{array}{l}\text { Ramirez et al. [58] } \\
(1997) /(1980-1993)\end{array}$ & $\begin{array}{l}2500 \\
(1000-4500)\end{array}$ & 30 & $\begin{array}{l}\text { Luque or Luque- } \\
\text { Galveston (23), CDI 6, } \\
\text { TSRH1; half autograft } \\
\text { and half allograft }\end{array}$ & - & - & - \\
\hline $\begin{array}{l}\text { Bellen et al. [6] } \\
(1993) /(1984-)\end{array}$ & $\begin{array}{l}2633 \\
(+/-1100)\end{array}$ & 47 & $\begin{array}{l}\text { Luque (12), Luque- } \\
\text { Galveston (22), } \\
\text { Hartshill (13), fusion to } \\
\text { pelvis } 38 \text { of } 47 \text {, local } \\
\text { bone graft and allograft }\end{array}$ & $2633 / 136=194$ & $84.5 \%$ & - \\
\hline $\begin{array}{l}\text { Noordeen et al. [54] } \\
(1999) /(1983-1993)\end{array}$ & 2977 & 48 & $\begin{array}{l}\text { Harrington system and } \\
\text { Harrington-Luque } \\
\text { system }\end{array}$ & $2977 / 13=229$ & $87 \%$ & - \\
\hline $\begin{array}{l}\text { Bentley et al. [8] } \\
(2001) /(1983-1996)\end{array}$ & $\begin{array}{l}3034 \\
(500-8700)\end{array}$ & 64 & $\begin{array}{l}\text { Luque, Harrington- } \\
\text { Luque, or Luque- } \\
\text { Galveston, local bone } \\
\text { graft only }\end{array}$ & $3034 / 13=233$ & - & - \\
\hline $\begin{array}{l}\text { Weimann et al. [70] } \\
(1983) /(1974-1978)\end{array}$ & $\begin{array}{l}3067 \\
(1830-4400)\end{array}$ & 24 & $\begin{array}{l}2 \text { Harrington } \\
\text { distraction rods; } \\
\text { autogenous iliac crest } \\
\text { bone grafts }\end{array}$ & $3067 / 12.8=240$ & - & - \\
\hline Gibson et al. [29] (1978) & 3132 & 10 & Harrington rod & - & - & - \\
\hline $\begin{array}{l}\text { Heller et al. [34] } \\
(2001) /(1992-1998)\end{array}$ & $\begin{array}{l}3373 \\
(800-8500)\end{array}$ & 31 & ISOLA system & $3373 / 16=211$ & - & - \\
\hline $\begin{array}{l}\text { Shapiro et al. [61] } \\
(1992) /(1980-1990)\end{array}$ & $\begin{array}{l}3640 \\
(+/-1905)\end{array}$ & 27 & $\begin{array}{l}\text { Luque or Harrington- } \\
\text { Luque, iliac crest } \\
\text { autograft ( } 7) \text {, allograft } \\
(20)\end{array}$ & $3640 / 13=280$ & - & - \\
\hline $\begin{array}{l}\text { Swank et al. [65] } \\
(1982) /(1967-1979)\end{array}$ & $\begin{array}{l}4064 \\
(3300-6200)\end{array}$ & 13 & $\begin{array}{l}7 / 11 \text { with } 2 \text { distraction } \\
\text { Harrington rods }\end{array}$ & $4064 / 15=271$ & - & - \\
\hline $\begin{array}{l}\text { Sakai et al. [60] } \\
(1977) /(1972-1979)\end{array}$ & 4400 & 6 & - & - & - & - \\
\hline
\end{tabular}

Information concerning entities

such as spinal muscular atrophy, myelomeningocoele, and anterior approaches alone

for selected neuromuscular disorders

Table 6 provides information from smaller numbers of studies. Blood loss in spinal muscular atrophy surgery is considerable but distinctly less than in DMD. In one group of 26 the mean EBL was $1,437 \mathrm{ml}$ with EBL/vertebral level $103 \mathrm{ml}$. Most reports on spinal fusion in myelomeningo- coele have been pooled with other neuromuscular disorders and reported in the studies listed in Table 5. One study on this entity showed mean EBL levels of $1,960 \mathrm{ml}$ for posterior fusion without instrumentation, $1,729 \mathrm{ml}$ for posterior fusion with Harrington rod stabilization, and 1,841 and $2,134 \mathrm{ml}$ for combined anterior fusion and posterior fusion with instrumentation. The use of improved anterior instrumentation for myelomenigocoele is shown by two studies using the Cotrel-Dubousset-Hopf instrumentation with a mean EBL of $800 \mathrm{ml}$ in 16 cases in 
Table 5 Blood loss data from studies on pooled neuromuscular disorders (anterior and posterior approaches). A anterior approach, $P$ posterior approach, $A P$ anterior and posterior approach, $C D I$ Cotrel-Dubousset instrumentation

\begin{tabular}{|c|c|c|c|c|c|c|}
\hline $\begin{array}{l}\text { Study [reference] } \\
\text { (date of publication) / } \\
\text { (date(s) of surgery) }\end{array}$ & $\begin{array}{l}\text { Estimated } \\
\text { blood loss } \\
(\text { EBL })(\mathrm{ml}) \\
{[\text { mean }} \\
(\text { range })]\end{array}$ & $\begin{array}{l}\text { Number } \\
\text { of pro- } \\
\text { cedures }\end{array}$ & Technique & $\begin{array}{l}\text { Estimated blood } \\
\text { loss / individual } \\
\text { vertebral level in } \\
\text { fusion }(\mathrm{ml}) \\
(\text { EBL/\# levels=) }\end{array}$ & $\begin{array}{l}\text { Estimated } \\
\text { blood loss / } \\
\text { total blood } \\
\text { volume }\end{array}$ & Comments \\
\hline \multirow{2}{*}{$\begin{array}{l}\text { Floman et al. [24] } \\
(1982) /(1972-1977)\end{array}$} & A 1033 & 73 & \multirow{2}{*}{$\begin{array}{l}\text { Multiple techniques. } \\
\text { Posterior procedure } \\
\text { performed } 2 \text { weeks } \\
\text { after anterior } \\
\text { procedure }\end{array}$} & - & - & \multirow{2}{*}{$\begin{array}{l}\text { EBL in Dwyer } \\
\text { procedures } 1250\end{array}$} \\
\hline & P 2200 & - & & - & - & \\
\hline $\begin{array}{l}\text { Neustadt et al. [51] } \\
(1992) /(1985-1988)\end{array}$ & $\begin{array}{l}\text { P } 1945 \\
(450-4500)\end{array}$ & 18 & $\begin{array}{l}\text { Posterior fusion to } \\
\text { pelvis with CDI }\end{array}$ & - & - & - \\
\hline \multirow[t]{2}{*}{$\begin{array}{l}\text { Benson et al. [7] } \\
(1998) /(1990-1994)\end{array}$} & $\begin{array}{l}\text { P 1684 } \\
(450-4000)\end{array}$ & P 38 & \multirow{2}{*}{$\begin{array}{l}\text { Luque-Galveston and } \\
39 / 50 \text { Anterior } \\
\text { discectomy } \\
\text { and fusion without } \\
\text { instrumentation }\end{array}$} & - & - & \multirow{2}{*}{$\begin{array}{l}43 \text { with allograft } \\
\text { only; hypotensive } \\
\text { anesthesia and } \\
\text { autologous blood } \\
\text { retrieval }\end{array}$} \\
\hline & $\begin{array}{l}\text { AP } 2329 \\
(550-6000)\end{array}$ & AP 12 & & - & - & \\
\hline \multirow{2}{*}{$\begin{array}{l}\text { Boachie-Adjei et al. } \\
{[12](1989) /} \\
(1979-1984)\end{array}$} & $\begin{array}{l}\text { A } 1100 \\
(300-2225)\end{array}$ & AP 11 & \multirow{2}{*}{$\begin{array}{l}\text { Luque-Galveston. } \\
\text { Anterior fusion } \\
\text { without instrumen- } \\
\text { tation; local bone } \\
\text { graft with allograft }\end{array}$} & A $100 / 7.4=149$ & - & \multirow[t]{2}{*}{-} \\
\hline & $\begin{array}{l}\text { P } 2639 \\
(270-8000)\end{array}$ & P 35 & & P $2639 / 15=176$ & - & \\
\hline \multirow[t]{2}{*}{$\begin{array}{l}\text { Ferguson et al. [22] } \\
(1996) /(1977-1991)\end{array}$} & $\begin{array}{l}\text { 1. A } 896 \\
\text { P } 3360\end{array}$ & $\begin{array}{l}1.29 \\
-\end{array}$ & $\begin{array}{l}\text { 1. Two stages } \\
\text { (anterior discectomy } \\
\text { and fusion) rib with } \\
\text { no instrumentation. } \\
\text { Posterior Luque- } \\
\text { Galveston }\end{array}$ & $\begin{array}{l}\text { 1. A } 896 / 8=112 \\
\text { P } 3360 / 15=224\end{array}$ & $\begin{array}{l}\text { 1. A } 28 \% \\
\text { P } 100.9 \%\end{array}$ & \multirow[t]{2}{*}{-} \\
\hline & 2. AP 2058 & 2. 16 & $\begin{array}{l}\text { 2. Both procedures } \\
\text { same day }\end{array}$ & 2. $2058 / 14.9=138$ & $2.83 \%$ & \\
\hline $\begin{array}{l}\text { Bell et al. [5] } \\
(1989) /(1983-1986)\end{array}$ & $\begin{array}{l}\text { P } 3500 \\
(800-11000)\end{array}$ & 34 & $\begin{array}{l}\text { Unit rod system T2- } \\
\text { pelvis. Local bone } \\
\text { graft only }\end{array}$ & - & - & - \\
\hline
\end{tabular}

one report and a value of only $539 \mathrm{ml}$ in 21 neuromuscular patients, 12 of whom had myelomeningocoele, in another.

\section{Key factors determining amount of blood loss during scoliosis surgery}

The material presented above clearly documents the extent of intra-operative blood loss during scoliosis surgery in both AIS and secondary scoliosis in patients with an underlying neuromuscular disorder. It is evident that blood loss is increased in patients with a neuromuscular diagnosis and an increasingly large number of vertebral levels included in the fusion. Studies assessing patients by the specific neuromuscular disorder demonstrate increasing losses as one moves from the cerebral palsy group, to spinal muscular atrophy and myelomenigocoele, and then to DMD which has the highest mean blood loss values. Posterior spine fusion procedures tend to lose more blood than anterior procedures, although most of this loss is due to the considerably larger number of vertebral levels fused in posterior approaches.

Several detailed papers have quantified the blood loss differences with statistical validation in relation to these matters. In one study neuromuscular patients had an almost seven times higher risk of losing greater than $50 \%$ of their estimated total blood volume during scoliosis surgery compared to idiopathic scoliosis patients when the extent of surgery (number of segments fused), age, weight, and pre-operative coagulation profile were controlled for statistically [19]. Another study showed that an underlying neuromuscular disease, lower body weight, and a higher number of vertebrae fused independently predicted a greater number of allogeneic red blood cell transfusions [47].

A large study of 319 patients operated between 1984 and 1993 clearly correlated the amount of bleeding with the disorder causing the scoliosis. The mean peri-operative bleeding was $9.8 \mathrm{ml} / \mathrm{kg}$ for idiopathic scoliosis (159 patients), $14.1 \mathrm{ml} / \mathrm{kg}$ for secondary scoliosis [including cerebral palsy (22 patients), myelomeningocoele (spina bifida) 
Table 6 Blood loss data from studies on spinal muscular atrophy and myelomeningocoele. $C$ - $D$-Hopf Cotrel-Dubousset-Hopf instrumentation, $H$-rod Harrington rod

\begin{tabular}{|c|c|c|c|c|c|c|}
\hline $\begin{array}{l}\text { Study [reference] } \\
\text { (date of publication)/ } \\
\text { (date(s) of surgery) }\end{array}$ & $\begin{array}{l}\text { Estimated } \\
\text { blood loss } \\
(\mathrm{EBL})(\mathrm{ml}) \\
{[\text { mean }} \\
\text { (range)] }\end{array}$ & $\begin{array}{l}\text { Number } \\
\text { of pro- } \\
\text { cedures }\end{array}$ & Technique & $\begin{array}{l}\text { Estimated blood } \\
\text { loss / individual } \\
\text { vertebral level in } \\
\text { fusion (ml) } \\
\text { (EBL/\#levels=) }\end{array}$ & $\begin{array}{l}\text { Estimated } \\
\text { blood loss/ } \\
\text { total blood } \\
\text { volume }\end{array}$ & Comments \\
\hline $\begin{array}{l}\text { Noordeen et al. [54] } \\
(1999) /(1983-1993)\end{array}$ & $\begin{array}{l}1437 \\
(350-3500)\end{array}$ & 26 & $\begin{array}{l}\text { Luque and } \\
\text { Harrington-Luque }\end{array}$ & $1437 / 14=103$ & - & $\begin{array}{l}\text { Spinal muscular } \\
\text { atrophy cases }\end{array}$ \\
\hline $\begin{array}{l}\text { Hopf et al. [35] } \\
(1997) /(1992-1994)\end{array}$ & $\begin{array}{l}800 \\
(350-2500)\end{array}$ & 16 & C-D-Hopf & $800 / 4.7=170$ & - & $\begin{array}{l}\text { Posterior fusion also } \\
\text { done in all myelo- } \\
\text { meningoceole patients }\end{array}$ \\
\hline \multirow[t]{3}{*}{$\begin{array}{l}\text { Osebold et al. [55] } \\
(1982) /(1960-1979)\end{array}$} & $\begin{array}{l}1960 \\
(550-3250)\end{array}$ & 13 & $\begin{array}{l}\text { Posterior fusion } \\
\text { without instrumen- } \\
\text { tation }\end{array}$ & $1960 / 9=218$ & - & \\
\hline & $\begin{array}{l}2134 \\
(245-4500)\end{array}$ & 3 & $\begin{array}{l}\text { Anterior fusion } \\
\text { without instrumen- } \\
\text { tation and posterior } \\
\text { H-rod fusion; } 2 \text { al- } \\
\text { lograft, } 1 \text { autograft }\end{array}$ & - & - & $\begin{array}{l}\text { Blood loss levels } \\
\text { for combined surgery, } \\
\text { not separated by site }\end{array}$ \\
\hline & $\begin{array}{l}1841 \\
(100-5200)\end{array}$ & $\begin{array}{l}17 \text { Patients } \\
\text { ( } 40 \text { proce- } \\
\text { dures) }\end{array}$ & $\begin{array}{l}\text { Anterior fusion with } \\
\text { Dwyer or Zielke; } \\
\text { posterior H-rod } \\
\text { fusion; } 10 \text { allograft, } \\
7 \text { autograft }\end{array}$ & - & - & \\
\hline
\end{tabular}

(18 patients) and vertebral malformations (18 patients)], and $29.3 \mathrm{ml} / \mathrm{kg}$ for muscular dystrophy and spinal muscular atrophy (31 patients) [23].

Other factors increasing blood loss are the length of time for the surgery to be done and the extra loss associated with harvesting autogenous iliac crest blood. In one study involving 145 patients undergoing Cotrel-Dubousset posterior fusions mean blood loss was $500 \mathrm{ml}$ at $2 \mathrm{~h}$, $1,500 \mathrm{ml}$ at $3 \mathrm{~h}$, and $2,400 \mathrm{ml}$ at $4.5 \mathrm{~h}$. The same study documented $1,828 \mathrm{ml}$ blood loss with autogenous iliac crest grafts and $1,120 \mathrm{ml}$ when autogenous bone was not used [57].

There are several reasons why blood loss is greater in the neuromuscular patients. Some are easy to understand and include: a larger number of vertebral levels fused, more frequent resort to both anterior and posterior procedures and the fact that the patients are often younger and of less weight than patients with idiopathic scoliosis. A recent study also documented a prolonged prothrombin time and decrease in factor VII activity greater than seen in idiopathic patients intra-operatively. The authors suggested that consumption of clotting factors during spinal surgery along with dilution of clotting factors further enhanced blood loss [38]. In addition, many neuromuscular patients are poorly nourished and have been on seizure medications, some of which can affect coagulation.

Awareness of the extent of blood loss with scoliosis surgery helps with pre-operative preparation and intraoperative management and should enhance the study and development of methods to decrease its occurrence. 


\section{References}

1. Albers HW, Hresko T, Carlson J, Hall JE (2000) Comparison of single- and dual-rod techniques for posterior spinal instrumentation in the treatments of adolescent idiopathic scoliosis. Spine 25:1944-1949

2. Allen BL, Ferguson RL (1982) L-rod instrumentation for scoliosis in cerebral palsy. J Pediatr Orthop 2:87-90

3. Barr SJ, Schuette AM, Emans JB (1997) Lumbar pedicle screws versus hooks: results in double major curves in adolescent idiopathic scoliosis. Spine 22:1369-1379

4. Basobas L, Mardjetko S, Hammerberg K, Lubicky J (2003) Selective anterior fusion and instrumentation for the treatment of neuromuscular scoliosis. Spine 28(20S):S245-248

5. Bell DF, Moseley CF, Koreska J (1989) Unit rod segmental spinal instrumentation in the management of patients with progressive neuromuscular spinal deformity. Spine 14:13011307

6. Bellen P, Hody JL, Clairbois J, Denis N, Soudon PH (1993) The surgical treatment of spinal deformities in Duchenne muscular dystrophy. J Orthop Surg 7:48-57

7. Benson ER, Thomson JD, Smith BG, Banta JV (1998) Results and morbidity in a consecutive series of patients undergoing spinal fusion for neuromuscular scoliosis. Spine 23:2308-2318

8. Bentley G, Haddad F, Bull TM, Seingry D (2001) The treatment of scoliosis in muscular dystrophy using modified Luque and Harrington-Luque instrumentation. J Bone Joint Surg 83B:2228

9. Bernstein RM, Hall JE (1998) Solid rod short segment anterior fusion in thoracolumbar scoliosis. J Pediatr Orthop B7:124-131

10. Betz RR, Harms J, Clements DH, Lenke LG, Lowe TG, Shufflebarger HL, Jeszenzsky S, Beele B (1999) Comparison of anterior and posterior instrumentation for correction of adolescent thoracic idiopathic scoliosis. Spine 24:225-239

11. Bitan FD, Neuwirth MG, Kuflik PL, Casden A, Bloom N, Siddiqui S (2002) The use of short and rigid anterior instrumentation in the treatment of idiopathic thoracolumbar scoliosis. Spine 27:1553-1557

12. Boachie-Adjei O, Lonstein JE, Winter RB, Koop S, Brink KV, Denis F (1989) Management of neuromuscular spinal deformities with Luque segmental instrumentation. J Bone Joint Surg 71A:548-562

13. Bonnett C, Brown JC, Grow T (1976) Thoracolumbar scoliosis in cerebral palsy. J Bone Joint Surg 58A:328-336
14. Bullman V, Halm HF, Nieyemer T, Hackenberg L, Liljenqvist U (2003) Dual-rod correction and instrumentation of idiopathic scoliosis with the Halm-Zielke instrumentation. Spine 28:1306-1313

15. Bulman WA, Dormans JP, Ecker ML, Drummond DS (1996) Posterior spinal fusion for scoliosis in patients with cerebral palsy: a comparison of Luque rod and unit rod instrumentation. J Pediatr Orthop 16:314-323

16. Copley LAB, Richards S, Safavi FZ, Newton PO (1999) Hemodilution as a method to reduce transfusion requirements in adolescent spine fusion surgery. Spine 24:219-224

17. Dias RD, Miller F, Dabney K, Lipton G, Temple T (1996) Surgical correction of spinal deformity using a rod in children with cerebral palsy. J Pediatr Orthop 16:734-740

18. Du Toit G, Relton JES, Gillespie R (1978) Acute haemodilutional autotransfusion in the surgical management of scoliosis. J Bone Joint Surg 60B: 178-180

19. Edler A, Murray DJ, Forbes RB (2003) Blood loss during posterior spinal fusion surgery in patients with neuromuscular disease: is there an increased risk? Paediatr Anaesth 13:818-822

20. Erwin WD, Dickson JH, Harrington PR (1976) The postoperative management of scoliosis patients treated with Harrington instrumentation and fusion. J Bone Joint Surg 58A:479-782

21. Feldman JM, Roth JV, Bjoraker DG (1995) Maximum blood savings by acute normovolemic hemodilution. Anesth Analg 80:108-113

22. Ferguson RL, Hansen MM, Nicholas DA, Allen BL (1996) Same-day versus staged anterior-posterior spinal surgery in a neuromuscular scoliosis population: the evaluation of medical complications. J Pediatr Orthop 16:193-303

23. Fievez E, Schultze-Balin C, Herbaux B, Dalmas S, Scherpereel P (1995) Etude du saignement dans la chirurgie de la scoliose. Interventions par voie posterieure chez 319 adolescents. Cah Anesthesiol 43:425-433

24. Floman Y, Micheli LJ, Penny JN, Riseborough EJ, Hall JE (1982) Combined anterior and posterior fusion in seventy-three spinally deformed patients: indications, results, and complications. Clin Orthop 164:110-121

25. Florentino-Pineda I, Blakemore LC, Thompson GH, Poe-Kochert C, Adler $P$, Tripi P (2001) The effect of $\varepsilon$-aminocaproic acid on perioperative blood loss in patients with idiopathic scoliosis undergoing posterior spinal fusion: a preliminary study. Spine 26 :

1147-1151
26. Florentino-Pineda I, Thompson GH, Poe-Kochert C, Huang RP, Haber LL, Blakemore LC (2004) The effect of amicar on perioperative blood loss in idiopathic scoliosis: the results of a prospective, randomized double blind study. Spine 29:233-238

27. Fox HJ, Thomas CH, Thompson AG (1997) Spinal instrumentation for Duchenne's muscular dystrophy: experience of hypotensive anaethesia to minimize blood loss. J Pediatr Orthop 17:750-753

28. Gersoff WK, Renshaw TS (1988) The treatment of scoliosis in cerebral palsy by posterior spinal fusion with Luquerod segmental instrumentation. J Bone Joint Surg 70A:41-44

29. Gibson DA, Koreska J, Robertson D, Kahn III A, Albisser AM (1978) The management of spinal deformity in Duchenne's muscular dystrophy. Orthop Clin North Am 9:437-450

30. Guadagni J, Drummond D, Breed A (1984) Improved postoperative course following modified segmental instrumentation and posterior spinal fusion for idiopathic scoliosis. J Pediatr Orthop 4:405:408

31. Guay J, Haig M, Lortie L, Guertin MC, Poitras B (1994) Predicting blood loss in surgery for idiopathic scoliosis. Can J Anaesth 41:775-781

32. Guidera KJ, Hooten J, Weatherly W, Highhouse M, Castellvi A, Ogden JA, Pugh L, Cook S (1993) Cotrel-Dubousset instrumentation: results in 52 patients. Spine 18:427-431

33. Harrington PR, Dickson JH (1973) An eleven-year clinical investigation of Harrington instrumentation. Clin Orthop 93:113-130

34. Heller KD, Wirtz DC, Siebert CH, Forst R (2001) Spinal stabilization in Duchenne muscular dystrophy: principles of treatment and record of 31 operative treated cases. J Pediatr Orthop 10:18-24

35. Hopf CG, Eysel P, Dubousset J (1997) Operative treatment of scoliosis with Cotrel-Dubousset-Hopf instrumentation. Spine 22:617-628

36. Hsu LCS, Sucherman J, Tang SC, Leong JCY (1982) Dwyer instrumentation in the treatment of adolescent idiopathic scoliosis. J Bone Joint Surg 64B:536-541

37. Kaneda K, Shono Y, Satoh S, Abumi K (1997) Anterior correction of thoracic scoliosis with Kaneda anterior spinal system: a preliminary report. Spine 22:1358-1368 
38. Kannan S, Meert KL, Mooney JF, Hillman-Wiseman C, Warrier I (2002) Bleeding and coagulation changes during spinal fusion surgery: a comparison of neuromuscular and idiopathic scoliosis patients. Pediatr Crit Care Med 3: 364-369

39. Lawhon SM, Kahn A, Crawford AH, Brinker MS (1984) Controlled hypotensive anesthesia during spinal surgery: a retrospective study. Spine 9: 450-453

40. Lenke LG, Bridwell K, Baldus C, Blanke K, Schoenecker PL (1992) Cotrel-Dubousset instrumentation for adolescent idiopathic scoliosis. J Bone Joint Surg 74A:1056-1067

41. Lonstein JE, Akbarnia BA (1983) Operative treatment of spinal deformities in patients with cerebral palsy or mental retardation. J Bone Joint Surg 65A:43-55

42. Lovallo JL, Banta JV, Renshaw TS (1986) Adolescent idiopathic scoliosis treated by Harrington-rod distraction and fusion. J Bone Joint Surg 68A: 1326-1330

43. Lowe TG, Peters JD (1993) Anterior spinal fusion with Zielke instrumentation for idiopathic scoliosis: a frontal and sagittal curve analysis in 36 patients. Spine 18:423-426

44. Majad ME, Castro FP, Holt RT (2000) Anterior fusion for idiopathic scoliosis. Spine 25:696-702

45. Marchesi D, Arlet V, Stricker U, Aebi M (1997) Modification of the original Luque technique in the treatment of Duchenne's neuromuscular scoliosis. J Pediatr Orthop 17:743-749

46. McMaster M (1991) Luque rod instrumentation in the treatment of adolescent idiopathic scoliosis: a comparative study with Harrington instrumentation. J Bone Joint Surg 73B:982-989

47. Meert KL, Kannan S, Mooney JF (2002) Predictors of red cell transfusion in children and adolescents undergoing spinal fusion surgery. Spine 27: 2137-2142

48. Moran MM, Kroon D, Tredwell SJ, Wadsworth LD (1995) The role of autologous blood transfusion in adolescents undergoing spinal surgery. Spine 20:532-536
49. Moskowitz A, Trommanhauser S (1993) Surgical and clinical results of scoliosis surgery using Zielke instrumentation. Spine 18:2444-2451

50. Mubarak SJ, Morin WD, Leach J (1993) Spinal fusion in Duchenne muscular dystrophy - fixation and fusion to the sacropelvis? J Pediatr Orthop 13: 752-757

51. Neustadt JB, Shufflebarger Hl, Cammisa FP (1992) Spinal fusion to the pelvis augmented by Cotrel-Dubousset instrumentation for neuromuscular scoliosis. J Pediatr Orthop 12:465-469

52. Newton PO, Marks M, Faro F, Betz R, Clements D, Haher T, Lenke L, Lowe T, Merola A, Wenger D (2003) Use of video-assisted thoracoscopic surgery to reduce perioperative morbidity in scoliosis surgery. Spine 28:249-254

53. Newton PO, Wenger DR, Mubarak SJ, Meyer RS (1997) Anterior release and fusion in pediatric spinal deformity: a comparison of early outcome and cost of thoracoscopic and open thoracotomy approaches. Spine 22:1398-1406

54. Noordeen MHH, Haddad FS, Muntoni F, Gobbi P, Hollyer JS, Bentley G (1999) Blood loss in Duchenne muscular dystrophy: vascular smooth muscle dysfunction? J Pediatr Orthop B8:212215

55. Osebold WR, Mayfield JK, Winter RB, Moe JH (1982) Surgical treatment of paralytic scoliosis associated with myelomeningocele. J Bone Joint Surg 64A:841-856

56. Patel NJ, Patel BS, Paskin S, Laufer S, Branch L (1985) Induced moderate hypotensive anesthesia for spinal fusion and Harrington-rod instrumentation. J Bone Joint Surg 67A:1384-1387

57. Pouliquen JC, Jean N, Noat M, Boyer J-M, Yannoutsos H (1990) Les economies de sang en orthopedie pediatrique. Etude a propos de 145 arthrodeses vertebrales posterieures. Chirurgie 116:303-311

58. Ramirez N, Richards BS, Warren PD, Williams GR (1997) Complications after posterior spinal fusion in Duchenne's muscular dystrophy. J Pediatr Orthop 17:109-114

59. Richards BS, Herring JA, Johnston CE, Birch JG, Roach JW (1993) Treatment of adolescent idiopathic scoliosis using Texas Scottish Rite Hospital instrumentation. Spine 19:1598-1605

60. Sakai DN, Hsu JD, Bonnett CA, Brown JC (1977) Stabilization of the collapsing spine in Duchenne muscular dystrophy. Clin Orthop 128:256-260
61. Shapiro F, Sethna N, Colan S, Wohl ME, Specht L (1992) Spinal fusion in Duchenne muscular dystrophy: a multidisciplinary approach. Muscle Nerve 15:604-614

62. Shufflebarger HL, Geck MJ, Clark CE (2004) The posterior approach for lumbar and thoracolumbar adolescent idiopathic scoliosis: posterior shortening and pedicle screws. Spine 29:269-276

63. Siller TA, Dickson JH, Erwin WD (1996) Efficacy and cost considerations of intraoperative autologous transfusion in spinal fusion for idiopathic scoliosis with predeposited blood. Spine 21:848-852

64. Sponseller PD, Whiffen JR, Drummond DS (1986) Interspinous process segmental spinal instrumentation for scoliosis in cerebral palsy. J Pediatr Orthop 6:559-563

65. Swank SM, Brown JC, Perry RE (1982) Spinal fusion in Duchenne's muscular dystrophy. Spine 7:484-491

66. Swank SM, Cohen DS, Brown JC (1989) Spine fusion in cerebral palsy with l-rod segmental spinal instrumentation: a comparison of single and twostage combined approach with Zielke instrumentation. Spine 14:750-758

67. Tate DE, Friedman RJ (1992) Blood conservation in spinal surgery. Review of current techniques. Spine 17:1450 1456

68. Theroux MC, Corddry DH, Tietz AE, Miller F, Peoples JD, Kettrick RG (1997) A study of desmopressin and blood loss during spinal fusion for neuromuscular scoliosis. Anesthesiology 87:260-267

69. Turi M, Johnston CE, Richards BS (1993) Anterior correction of idiopathic scoliosis using TSRH instrumentation. Spine 18:417-422

70. Weimann RL, Gibson DA, Moseley CF, Jones DC (1983) Surgical stabilization of the spine in Duchenne muscular dystrophy. Spine 8:776-780

71. Youngman PME, Edgar MA (1985) Posterior spinal fusion and instrumentation in the treatment of adolescent idiopathic scoliosis. Ann R Coll Surg Engl 67:313-317 